\title{
El manejo de la presión arterial en la era post-SPRINT
}

The management of blood pressure in the post- SPRINT era

Lisandro Colantonio y María Soledad Burrone $^{\S}$

Colantonio L, Burrone MS. El manejo de la presión arterial en la era post-SPRINT. Evid Act Pract Ambul. 2015;18(4):104-105. Oct-Dic.

En septiembre de 2015, representantes de los Institutos de Salud de los Estados Unidos anunciaron que terminaban en forma anticipada el estudio SPRINT (del inglés Systolic Blood Pressure Intervention Trial) por encontrar diferencias estadísticamente significativas en los resultados analizados. El estudio SPRINT había incluido adultos de 50 años de edad o mayores, con presión arterial sistólica (PAS) entre 130 y $180 \mathrm{mmHg}$ y con riesgo cardiovascular alto, definido por presentar antecedentes de enfermedad cardiovascular, enfermedad renal crónica con filtrado glomerular de 20 a $60 \mathrm{~mL} / \mathrm{min} / 1,73 \mathrm{~m}^{2}$, más de $15 \%$ de riesgo cardiovascular a diez años usando las ecuaciones Framingham, o edad mayor a 75 años'. Fueron excluidos los adultos con diabetes, enfermedad cerebrovascular, proteinuria, insuficiencia cardíaca o insuficiencia renal crónica con filtrado glomerular menor de $20 \mathrm{~mL} / \mathrm{min} / 1,73 \mathrm{~m}^{2}$ o en diálisis.

Los participantes del estudio SPRINT fueron aleatorizados a recibir un manejo intensivo de la PAS (con un objetivo menor a $120 \mathrm{mmHg}$ ) o un manejo estándar (PAS menor a $140 \mathrm{mmHg}$ ). Los resultados de interés del estudio fueron la ocurrencia de eventos cardiovasculares (ej. infarto de miocardio, síndrome coronario agudo, accidente cerebrovascular, insuficiencia cardíaca descompensada o muerte cardiovascular) y muerte por cualquier causa. La decisión de terminar el estudio se tomó luego de que dos análisis interinos consecutivos mostraron que la estrategia de manejo intensivo de la PAS se asoció a una reducción del $25 \%$ del riesgo de eventos cardiovasculares (RR: $0,75 ; \mathrm{IC} 95 \% 0,64$ a 0,89$)$ y $27 \%$ del riesgo de muerte $(0,73$; $0,60$ a 0,90$)$, en comparación con el manejo estándar².

Los responsables de elaborar y actualizar las guías para el manejo de la presión arterial, y los médicos en general, deberán considerar como incorporar los resultados del estudio SPRINT en la práctica clínica. En principio, parece razonable que se recomiende un manejo intensivo de la PAS en aquellos que cumplen con los criterios de inclusión y exclusión del estudio SPRINT. Esto implicaría un cambio substancial en el manejo de la presión arterial a nivel poblacional. En EE.UU. se estima que un $17,6 \%$ de los adultos de 50 años o más (alrededor de 16,8 millones de personas) cumplen con los criterios de inclusión y exclusión del estudio SPRINT, y que la mitad de ellos no está recibiendo tratamiento antihipertensivo ${ }^{3}$.

El cambio más significativo en el manejo de la presión arterial podría verse para los adultos mayores, quienes, de acuerdo a las guías de práctica clínica vigentes, en general tienen objetivos de PAS más altos que los adultos jóvenes. Por ejemplo, las guías y los consensos para el manejo de la presión arterial en Argentina y Europa recomiendan un objetivo terapéutico de PAS menor a $150 \mathrm{mmHg}$ en adultos de más de 80 años de edad $^{4-6}$. En EE.UU. los miembros del octavo Comité Conjunto para la Prevención, Detección, Evaluación y Tratamiento de la Presión Arterial Alta recomendaron en 2014 elevar el objetivo terapéutico de PAS en adultos de 60 años o mayores desde menos de $140 \mathrm{mmHg}$ hacia menos de $150 \mathrm{mmHg}^{7}$. Cuando el análisis se limitó a participantes de 75 años de edad o mayores, los resultados del estudio SPRINT informan que el manejo intensivo de la PAS se asoció con una reducción del riesgo de eventos cardiovasculares y de muerte de un $33 \%$
(RR 0,$67 ; 0,51$ a 0,86$)$ y $32 \%(0,68 ; 0,50$ a 0,92$)$, respectivamente. De acuerdo a los resultados de SPRINT, todos los adultos mayores de 75 años que no cumplen con los criterios de exclusión de este estudio podrían ser candidatos para un manejo intensivo de la PAS.

Los encargados de revisar las guías de práctica clínica deberán analizar si se debería recomendar un manejo intensivo de la PAS en personas que fueron excluidas del estudio SPRINT, como por ejemplo aquellos con diabetes, enfermedad cerebrovascular, menores de 50 años, con PAS entre 120 y $129 \mathrm{mmHg}$ o con riesgo cardiovascular bajo o moderado. Aunque sería deseable que se realicen estudios sobre la eficacia del manejo intensivo de la PAS en estas poblaciones, es poco probable que esto ocurra en el corto plazo. SPRINT excluyó participantes con diabetes dado que esta población fue analizada en el estudio ACCORD (del inglés Action to Control Cardiovascular Risk in Diabetes) ${ }^{8}$. En este estudio, el manejo intensivo de la PAS (menor a $120 \mathrm{mmHg}$ ) se asoció con una reducción estadísticamente no significativa de un $12 \%$ del riesgo de eventos cardiovasculares (RR 0,88; 0,73 a 1,06) en comparación con el manejo estándar (PAS menor a $140 \mathrm{mmHg}$ ) 9 . Sin embargo, el estudio ACCORD había incluido la mitad del número de participantes en comparación con el estudio SPRINT, lo que deriva en un menor poder estadístico. Además, el estudio ACCORD no analizó la ocurrencia de insuficiencia cardíaca descompensada, uno de los resultados que más contribuyó al efecto global del manejo intensivo de la PAS en el estudio SPRINT. Finalmente, se ha propuesto que la ausencia de una diferencia significativa a favor del manejo intensivo de la PAS en el estudio ACCORD puede deberse a una interacción con el manejo intensivo de la glucemia que se realizó en forma concomitante en muchos participantes ${ }^{10}$. Teniendo en cuenta estas limitaciones, resulta significativo destacar que el IC95\% para la eficacia del manejo intensivo de la PAS en ACCORD no descarta la posibilidad de una reducción de hasta un $27 \%$ del riesgo cardiovascular en personas con diabetes, similar a la observada en estudio SPRINT ${ }^{11}$.

Los adultos con antecedentes de enfermedad cerebrovascular también podrían beneficiarse de un manejo intensivo de la PAS. El estudio SPS3 (del inglés Secondary Prevention of Small Subcortical Strokes), investigó la eficacia de un manejo intensivo de presión arterial (definido por establecer como meta una PAS menor a $130 \mathrm{mmHg}$ ) comparado con un manejo estándar (PAS 130 a $149 \mathrm{mmHg}$ ) en personas con un evento cerebrovascular reciente, caracterizado como infarto lacunar. En este estudio, el manejo intensivo de la PAS se asoció con una reducción del $19 \%$ del riesgo de eventos cerebrovasculares (RR 0,$81 ; 0,64$ a 1,03) y del $16 \%$ de infarto de miocardio o muerte (RR 0,84; 0,68 a 1,04), ambas no estadísticamente significativas $^{12}$. Los autores de este estudio, que había incluido un tercio del número de participantes que la cantidad incluida en el estudio SPRINT, concluyeron que es probable que sea beneficioso reducir la PAS a menos de $130 \mathrm{mmHg}$ en personas con el antecedente reciente de un accidente cerebrovascular lacunar. Análisis subsecuentes del estudio SPS3 mostraron además, que el manejo intensivo de la PAS no se asoció con un aumento del riesgo de eventos adversos ${ }^{13}$.

₹ Department of Epidemiology. University of Alabama at Birmingham, Birmingham, Alabama, Estados Unidos. Escuela de Salud Pública, Facultad de Medicina de la Universidad de Buenos Aires, Buenos Aires, Argentina. lisandro.colantonio@fulbrightmail.org. 
Si bien el estudio SPRINT incluyó participantes con riesgo cardiovascular alto, sus resultados también podrían generalizarse a personas con menor riesgo, incluyendo aquellos menores de 50 años de edad o con PAS entre 120 y $129 \mathrm{mmHg}$. En general, los ensayos clínicos incluyen participantes de alto riesgo para el resultado primario por razones de eficiencia. Específicamente, incluir personas de alto riesgo garantiza un número de eventos alto, lo cual conlleva que el estudio pueda realizarse en menor tiempo, con menos participantes y a menor costo. Aunque las personas menores de 50 años de edad, con PAS 120 a 129 mmHg o con riesgo cardiovascular bajo o moderado quizás podrían beneficiarse de un manejo intensivo de la PAS, la reducción del riesgo absoluto será sin duda menor que en poblaciones de mayor riesgo. Por lo tanto, sería necesario conducir estudios de riesgo-beneficio y costo-efectividad para informar la decisión de recomendar un manejo intensivo de la PAS en estas poblaciones. Sin embargo, el impacto poblacional de recomendar un manejo intensivo de la PAS en personas de bajo riesgo cardiovascular sería substancial. Por ejemplo, se estima que el $38,6 \%$ de la población de EE.UU. mayor de 20 años de edad (alrededor de 84,7 millones de personas) tienen PAS igual o mayor a $120 \mathrm{mmHg}$ y no cumplen con ningún criterio de exclusión de SPRINT ${ }^{3}$.

En el estudio SPRINT, el manejo intensivo de la PAS se asoció con un riesgo mayor de hipotensión $(2,4 \%$ para el manejo intensivo versus $1,4 \%$ para el manejo estándar, p: 0,001), síncope $(2,3 \%$ versus $1,7 \%, p: 0.05)$, alteraciones electrolíticas $(3,1 \%$ versus $2,3 \%, p: 0.02)$ y daño renal agudo $(4,1 \%$ versus $2,5 \%, p<0,001)^{2}$. En cambio, no se observó un aumento en el riesgo de caídas que condujeran a una evaluación en sala de emergencias u hospitalización (2,2\% para el manejo intensivo versus $2,3 \%$ para el manejo estándar, p: 0,71 ) o eventos adversos graves totales $(38,3 \%$ versus $37,1 \%, p: 0,25)$. Es importante mencionar que la evaluación de eventos adversos en el estudio SPRINT pudo estar sesgada a favor del manejo estándar. A diferencia de la evaluación del resultado principal, que fue realizado en cuatro visitas anuales y por personal que desconocía el esquema de manejo asignado, la evaluación de los eventos adversos se realizó por parte del médico tratante (no ciego) y en cualquier visita ${ }^{11}$.

Los individuos asignados al manejo intensivo de la PAS en el estudio SPRINT requirieron un 20 a un $30 \%$ más visitas que los asignados al manejo estándar, lo que brinda más oportunidades para reportar eventos adversos.

$\mathrm{Si}$ bien el mayor riesgo de eventos adversos asociado con el manejo intensivo de la PAS en participantes del estudio SPRINT parece ser aceptable en relación con la reducción del riesgo de eventos cardiovasculares y muerte, esto podría no ser así en otras poblaciones, como aquellos más jóvenes o con mayor riesgo de hipotensión. Por ello, es importante que las guías de manejo de la presión arterial y los médicos en general tengan en cuenta los potenciales efectos adversos así como las preferencias de los pacientes a la hora de recomendar un manejo intensivo de la PAS, especialmente en aquellas poblaciones que no fueron incluidas en el estudio SPRINT.

Otro cambio que se podría esperar en futuras guías y recomendaciones para el manejo de la presión arterial está relacionado con la forma en la que se identificarán los individuos que pueden ser candidatos para un manejo intensivo de la PAS. En el estudio SPRINT, los investigadores utilizaron las ecuaciones Framingham con el fin de identificar individuos con riesgo cardiovascular alto en la población de menores de 75 años sin antecedentes de enfermedad cardiovascular o enfermedad renal crónica ${ }^{1}$. Es posible que en el futuro, la mayoría de las guías de manejo de la presión arterial incorporen el uso de calculadoras de riesgo para guiar la decisión de iniciar tratamiento farmacológico, de forma similar a las guías de hipertensión del Instituto Nacional de Salud y Excelencia Clínica de Inglaterra ${ }^{14}$.

Para finalizar, es importante mencionar que aproximadamente la mitad de los participantes asignados a un manejo intensivo de PAS en los estudios SPRINT y ACCORD no alcanzaron el valor objetivo de llevar su PAS a menos de $120 \mathrm{mmHg}^{2,9}$. Esto demuestra la dificultad que se tendrá para alcanzar estos valores de PAS en la práctica clínica habitual, una cuestión que necesariamente deberá ser abordada en las guías de manejo de presión arterial para minimizar la frustración y ansiedad que puede generar en los pacientes no alcanzar sus metas terapéuticas. En resumen, los resultados del estudio SPRINT contribuirán a cambiar en forma substancial el manejo de la presión arterial a nivel poblacional. Resta por conocer los detalles de cómo se implementarán estos cambios en la práctica clínica habitual.

Conflictos de interés: Los autores declaran no tener conflictos de interés para reportar.

Recibido el 03/02/16 y aceptado el 06/02/16.

\section{Referencias}

1. Ambrosius W y col. The design and rationale of a multicenter clinical trial comparing two strategies for control of systolic blood pressure: the Systolic Blood Pressure Intervention Trial (SPRINT). Clinical trials (London, England) 2014;11(5):532-46.

2. Wright J y col. A Randomized Trial of Intensive versus Standard Blood-Pressure Control. The New England joumal of medicine 2015;373(22):2103-16.

3. Bress A y col. Generalizability of results from the Systolic Blood Pressure Intervention Trial (SPRINT) to the US adult population. Joumal of the American College of Cardiology 2015.

4. Guías de la Sociedad Argentina de Hipertensión Arterial para el diagnóstico, estudio, tratamiento y seguimiento de la hipertensión arterial. Buenos Aires: Sociedad Argentina de Hipertensión Arterial, 2011.

5. Consenso de Hipertensión Arterial. Revista Argentina de Cardiología 2013;81(Supl 2):7-78.

6. Mancia G y col. 2013 ESH/ESC Guidelines for the management of arterial hypertension: the Task Force for the management of arterial hypertension of the European Society of Hypertension (ESH) and of the European Society of Cardiology (ESC). J Hypertens 2013;31(7):1281-357.

7. James P y col. 2014 evidence-based guideline for the management of high blood pressure in adults: report from the panel members appointed to the Eighth Joint National Committee (JNC 8). JAMA : the journal of the American Medical Association 2014;311(5):507-20.

8. Goff $D$ y col. Prevention of cardiovascular disease in persons with type 2 diabetes mellitus: current knowledge and rationale for the Action to Control Cardiovascular Risk in Diabetes (ACCORD) trial. The American journal of cardiology 2007;99(12A):4i-20i.

9. Cushman W y col. Effects of intensive blood-pressure control in type 2 diabetes mellitus. The New England joumal of medicine 2010;362(17):1575-85.

10. Margolis $\mathrm{K}$ y col. Outcomes of combined cardiovascular risk factor management strategies in type 2 diabetes: the ACCORD randomized trial. Diabetes Care 2014;37(6):1721-8.

11. Cushman W y col. SPRINT Trial Results: Latest News in Hypertension Management. Hypertension 2016;67(2):263-5.

12. Benavente Oy col. Blood-pressure targets in patients with recent lacunar stroke: the SPS3 randomised trial. Lancet 2013;382(9891):507-15.

13. White C y col. Can blood pressure be lowered safely in older adults with lacunar stroke? The Secondary Prevention of Small Subcortical Strokes study experience. J Am Geriatr Soc 2015;63(4):722-9.

14. McCormack T y col. Management of hypertension in adults in primary care: NICE guideline. The British joumal of general practice : the joumal of the Royal College of General Practitioners 2012;62(596):163-4. 